\title{
World Health Organization type B2 thymoma with an abundance of Hassall's corpuscles: A case report
}

\author{
LI GONG ${ }^{1}$, LU SUN ${ }^{2}$, CHENLING ZHANG $^{3}$, SANHUA WEI $^{4}$, JUN CHEN $^{5}$, YAZHUO $^{2}{ }^{2}$, \\ YANHONG $\mathrm{LI}^{1}$, WEI ZHANG ${ }^{1}$ and YONGSHENG ZHANG ${ }^{6}$ \\ ${ }^{1}$ Department of Pathology, Tangdu Hospital, The Fourth Military Medical University, Xi'an, Shaanxi 710038; \\ ${ }^{2}$ Department of Pathology, Hainan Branch of General Hospital of The Chinese People's Liberation Army, Sanya, Hainan \\ 572013; ${ }^{3}$ Department of Gynaecology and Obstetrics, Baoji Central Hospital, Baoji, Shaanxi 721008; Departments of \\ ${ }^{4}$ Clinical Laboratory and ${ }^{5}$ Orthopedics, ${ }^{6}$ Research Service Office, Tangdu Hospital, The Fourth Military Medical University, \\ Xi'an, Shaanxi 710038, P.R. China
}

Received September 17, 2015; Accepted November 10, 2017

DOI: $10.3892 / 01.2018 .8459$

\begin{abstract}
Hassall's corpuscles (HCs) are characteristic components of the medulla of the mammalian thymus and thymoma with an abundance of HCs is even more rare, with only five cases having been reported in the literature, to the best of our knowledge. Furthermore, their morphology usually resembles World Health Organization (WHO) type B2 or B3 thymoma. The biological behavior appears indolent and is rarely associated with myasthenia gravis. However, whether it is a separate entity or a variant of organotypical thymoma WHO B2 or B3 remains to be elucidated. The present case report reports a type $\mathrm{B} 2$ thymoma with a large presence of $\mathrm{HCs}$ from a 58-year-old Chinese woman, and investigates its histopathological characteristics, immunological features and biological behavior. Notably, the present case appeared biologically more aggressive compared with the cases reported previously. The aim of describing this rare case of thymoma with an abundance of HCs is so that it can be recognized and classified by pathologists.
\end{abstract}

\section{Introduction}

Hassall's corpuscles (HCs) are characteristic structures of the normal thymus and are commonly used as diagnostic features for identifying the human thymus (1). The majority of studies consider that they are derived from epithelial cells of the thymic medulla (2-4). Typical HCs are occasionally identified in areas

Correspondence to: Professor Wei Zhang, Department of Pathology, Tangdu Hospital, The Fourth Military Medical University, 569 Xinsi Road, Baqiao, Xi'an, Shaanxi 710038, P.R. China

E-mail: zhwlyh@fmmu.edu.cn

Abbreviations: CK, cytokeratin; CT, computed tomography; EMA, epithelial membrane antigen; HCs, Hassall's corpuscles

Key words: Hassall's corpuscles, thymoma, biological behavior with medullary differentiation of World Health Organization (WHO) type B1-3 thymoma; however, they are not identified in type A and AB thymoma (5-11). Thymoma with an abundance of HCs is rare, with only five cases having been reported in the literature (12), to the best of our knowledge. The present case report concerns a type B2 thymoma with an abundance of HCs in a 58-year-old Chinese woman and considers its clinicopathological characteristics, immunological phenotype and biological behavior. The aim of describing this rare type of thymoma is so that it can be recognized and classified by pathologists. In addition, it is advised that clinical doctors should follow-up these patients due to its undefined biological behavior.

\section{Case report}

A 58-year-old Chinese woman was admitted to the Hainan Branch of General Hospital of the Chinese People's Liberation Army (Hainan, China) on 19 April 2015 due to a repeated pain in the right anterior chest for 6 months. The pain was often exacerbated when the body posture was changed and was accompanied simultaneously by malaise, without eyelid ptosis, cough, headache, shortness of breath or dizziness. A computed tomography (CT) scan of the chest revealed a heterogeneous mass, with a size of $8.2 \times 6.2 \times 4.6 \mathrm{~cm}$ in the right anterior superior mediastinum, involving the right pericardium and wrapping around the superior and inferior vena cava (Fig. 1). Thus, an exploratory thoracotomy was performed. However, it was not possible to resect the tumor completely as it was difficult to separate it from the superior and inferior vena cava. Grossly, the tumor adhered to the down lobe of the right lung, the size was $7.5 \times 7.5 \times 5 \mathrm{~cm}$, the encapsulation was not intact, the cut surface was yellow-grey and the texture was of a medium hardness. The resected samples were fixed in $10 \%$ neutral formalin solution, and embedded in paraffin. Serial sections were stained with hematoxylin and eosin (H\&E). Microscopically, a large quantity of HCs with various morphologies, including solid, cystic, round and irregular, were initially identified to be diffusely distributed. The size of the HCs varied from extremely small to large. Large and 
A

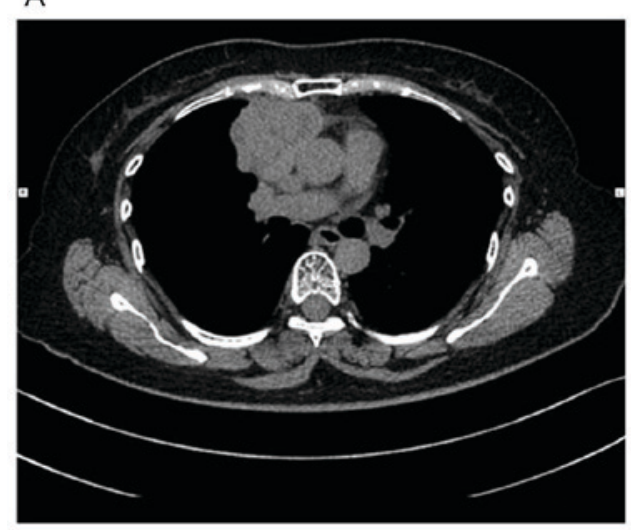

c

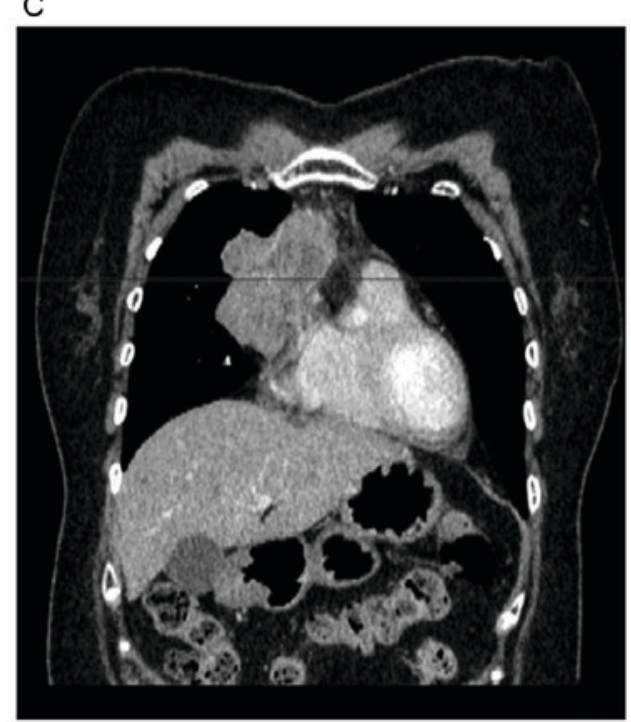

B

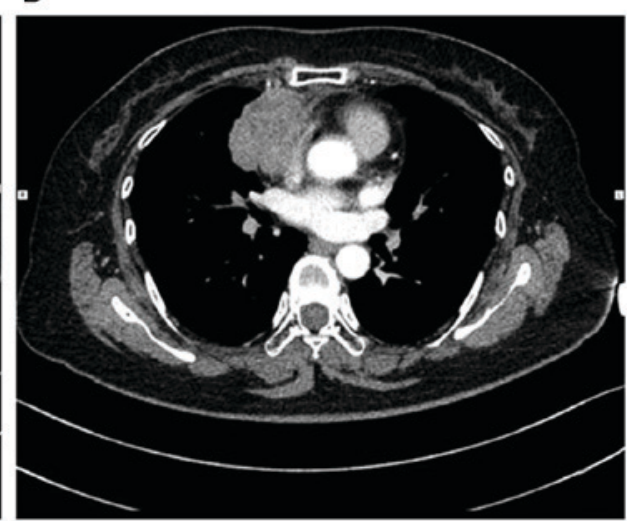

D

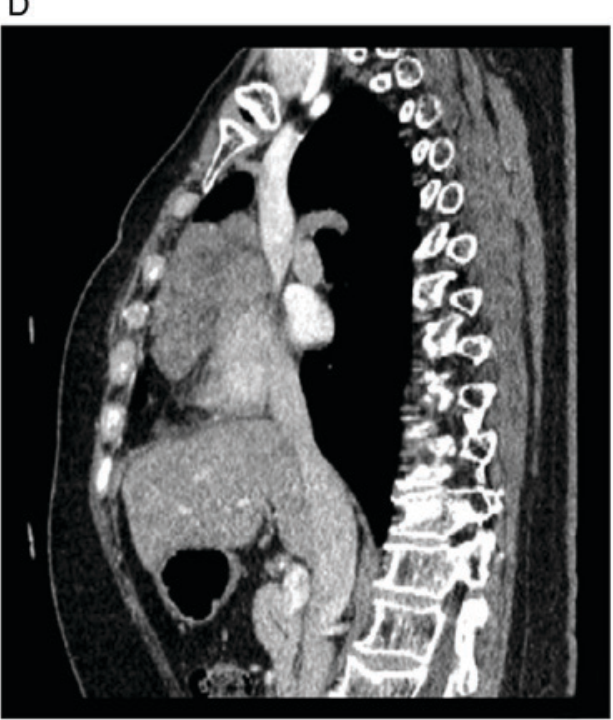

Figure 1. (A) Conventional and (B) enhanced computer tomography scans of the patient's chest revealed a heterogeneous mass of $8.2 \times 6.2 \times 4.6 \mathrm{~cm}$ in the right anterior superior mediastinum. (C) Coronal and (D) sagittal views showed the mass involved the right pericardium and wrapping around the superior and inferior vena cava.

polygonal neoplastic epithelial cells were scattered individually or in small clusters among immature lymphocytes, and formed a delicate loose network. Their nucleoli were prominent. Only a limited number of the tumor cells were arranged in the small papillary or nested histoarchitecture in the focal area. Certain tumor cells were involved in the lung tissues and adipose tissue out of the pleura. Furthermore, the HCs in the tumor involved in the lung tissues appeared to be small, and there were no cystic structures. Cystic spaces filled with fibrin, blood or HCs, cholesterol crystal, scarring, necrosis and calcium deposits were identified (Fig. 2). Immunohistochemically, Sections (4- $\mu \mathrm{m}$ thick) from a representative block were deparaffinized in $99 \%$ xylene for $15 \mathrm{~min}$ at room temperature, rehydrated in graded alcohol (ethanol) with 100, 95, 80 and 75\% in turn, incubated with $3 \% \mathrm{H}_{2} \mathrm{O}_{2}$ to block the activity of endogenous peroxidases for $10 \mathrm{~min}$ at room temperature. The sections were then subjected to heat-induced antigen retrieval in $0.1 \mathrm{~mol} / \mathrm{l}$ citrate buffer at $\mathrm{pH} 6.0$ for 3 min following treatment with EDTA buffer at pH 9.0 in a microwave for $15 \mathrm{~min}$. The slides were then incubated with ready-to-use mouse anti-human primary monoclonal antibody against pan-cytokeratin (CK; MAB-0671), CK19 (kit-0020), cluster of differentiation (CD)99 (MAB-0059), CD20 (MAB-0669), and CD1a (MAB-0336), rabbit anti-human monoclonal antibodies against epithelial membrane antigen (EMA) (kit-0011), CD5 (MAB-0252), and $\mathrm{CD} 117$ (MAB-0590) for $1 \mathrm{~h}$ at $37^{\circ} \mathrm{C}$. After excess primary antibody was removed with PBS buffer solution, the ready-to-use goat anti-mouse/rabbit IgG polymer conjugated with horseradish peroxidase (kit-0016) were applied and incubated for $20 \mathrm{~min}$ at room temperature. Then, a subsequent reaction was performed and visualized using $40 \mu 1$ 3.3'-diaminobenzidine (DAB) buffer with fresh configuration (concentration, $0.2 \mathrm{~g} / 20 \mathrm{ml} \mathrm{PBS}$ ) for 5-8 min at room temperature. Finally, the slides were incubated with hematoxylin staining solution for $20 \mathrm{sec}$, and washed using water. All of these reagents were provided by Maxim Biotech, Develop Co. (Fuzhou, Fujian, China). After mounting, the slides were observed by a light microscope (DM2500, Leica Microsystems Wetzlar GmbH). The results demonstrated that the tumor cells were positive for pan-CK and CK19, and negative for EMA and CD5, CD117 and CD20 (Fig. 3). Intraepithelial lymphocytes were positive for CD1a, CD5 and CD99. The index of Ki-67 was high ( $80 \%)$. Therefore, type B2 thymoma with an abundance of HCs was eventually diagnosed. However, she did not undergo further therapy and was discharged from the hospital for financial reasons. Therefore, the patient was not followed-up until she 


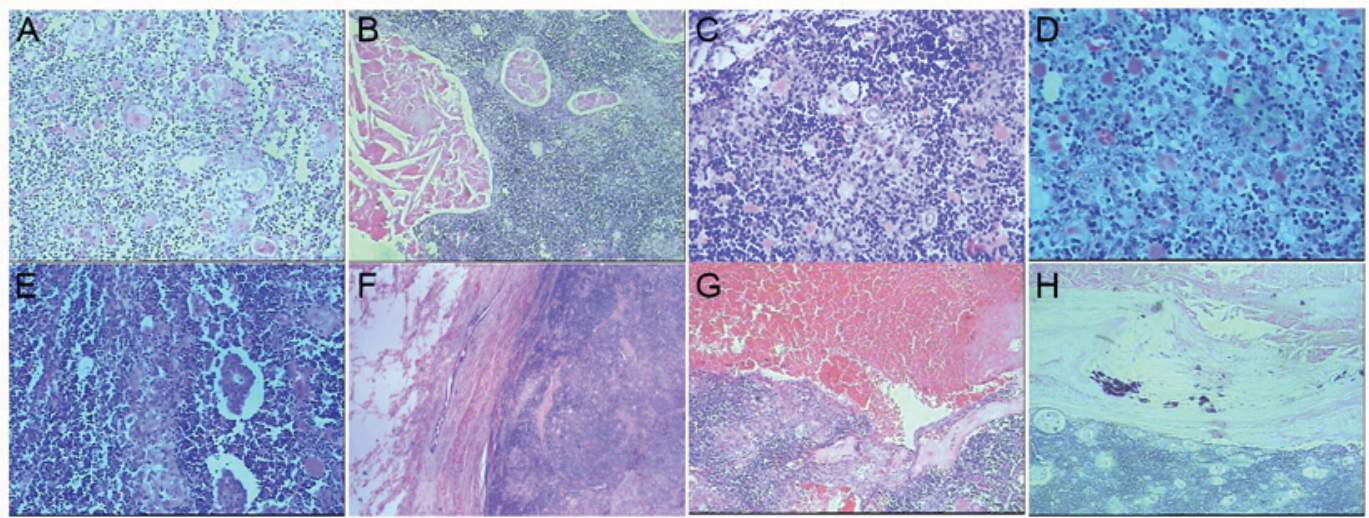

Figure 2. Microscopically, large HCs with various shapes, including solid, cystic, round and irregular HCs, were found to diffusely distribute. The size of HCs varied from (A) very small (magnification, x200) to (B) very large (magnification, x100). (C, magnification, x400) Large and polygonal neoplastic epithelial cells scattered individually or in small clusters among immature lymphocytes, and (D, magnification, $\mathrm{x} 400)$ formed a delicate loose network with prominent nucleoli. (E) Only a limited number of tumor cells were arranged in small papillary or nested histoarchitecture in focal area (magnification, x200). (F) Certain tumor cells were involved in the lung tissues (magnification, x100). Cystic spaces filled with (G) fibrin, blood or HCs (magnification, x100). (H) Cholesterol crystal, scarring, necrosis and calcium deposits were identified (magnification, x100).
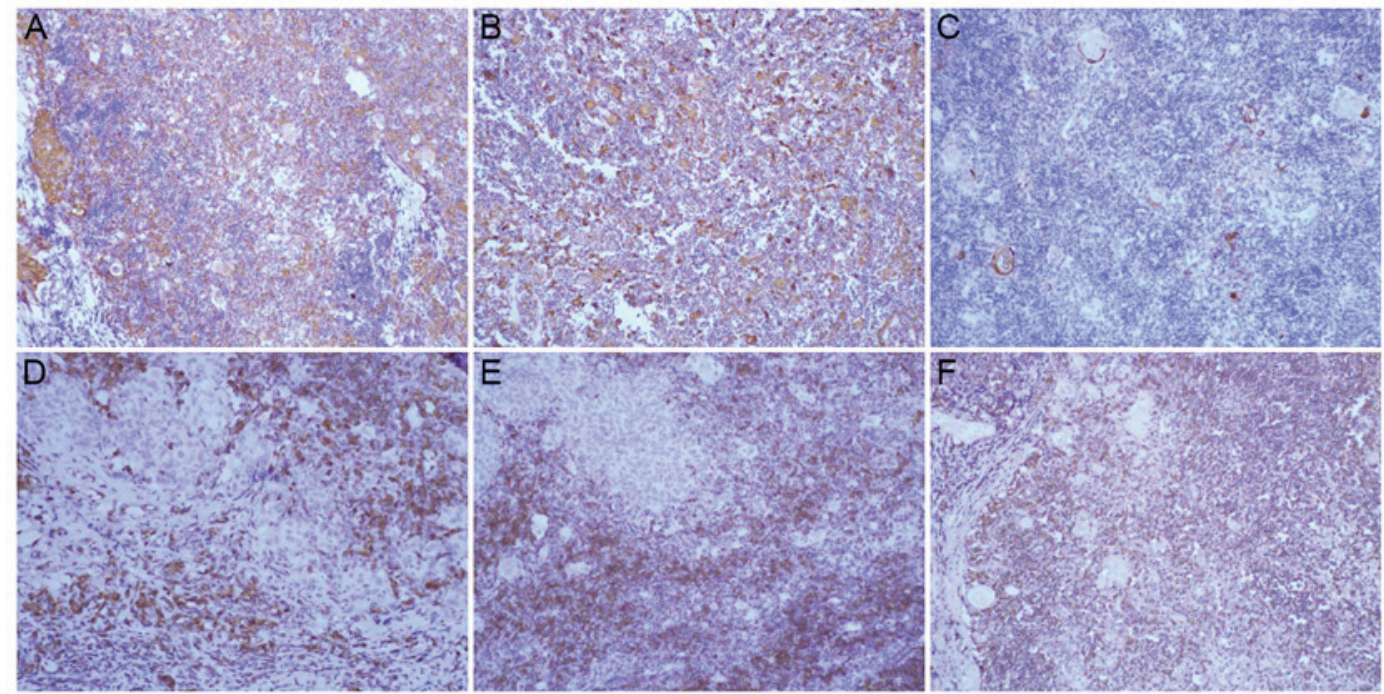

Figure 3. Immunohistochemistry. The tumor cells demonstrated positive expression of (A) CK19 (magnification, x100) and (B) pan CK (magnification, x100) and negative for (C) epithelial membrane antigen (magnification, x100). Intraepithelial lymphocytes demonstrated positive expression of (D) CD1a (magnification, x200), (E) CD5 (magnification, x200) and (F) CD99 (magnification, x200)

revisited due to pain in the right anterior chest 9 months after surgery. Unfortunately, the patient was unwilling to undergo an imaging examination again.

\section{Discussion}

HCs, which are characteristic structures of the human thymus, are derived from epithelial cells of the thymic medulla (2-4). The physical nature of these structures differs between mammalian species, for example, they are abundant in the human thymus, but relatively rare in the mouse thymus (13). It has been suggested previously that HCs serve a function in the removal of apoptotic thymocytes and the maturation of developing thymocytes within the thymus (14). However, the function remains to be elucidated. Typical HCs are occasionally identified in areas with medullary differentiation of WHO type B1-3 thymoma; however, they have not been identified in type $\mathrm{A}$ and $\mathrm{AB}$ thymoma (5-11). Thymoma with an abundance of HCs is rare, and has been termed 'corpuscular thymoma' by Laeng et al (12). To the best of our knowledge, only five cases have been reported previously (12). Clinically, the occurrence of the majority of thymoma are latent and not easily observed. Only one case demonstrated the symptom of eyelid ptosis. The other four cases were asymptomatic and were incidentally identified. Simultaneously, no association with myasthenia gravis or other autoimmune diseases was observed. The patient in the present study exhibited none of the aforementioned symptoms or diseases, with the exception of a repeated pain in the right anterior chest. The age range of the known cases was between 41 and 86 years, including the present case, and the average age was 64 years when the diagnosis was performed. The cases reported were of three men and three women, suggesting that sex is not a factor. 
Histopathologically, the morphology varied, although they resembled type B2 or B3 thymoma (12). In the present case, the majority of the epithelial cells formed a loose network and only a limited number of tumor cells were arranged in a small papillary or nested histoarchitecture in the focal area. The HCs were polymorphic, including solid, cystic, round, and irregular shapes. The size of HCs varied from extremely small to large. Cystic spaces, which were filled with fibrin, blood or HCs, were also observed. Immunohistochemically, the tumor cells were positive for CK19 and pan-CK with reticular formation, and negative for EMA, CD5, CD117 and CD20. These characteristics differed from those of type B3 thymoma or squamous cell carcinoma. In addition, intraepithelial lymphocytes were positive for CD1a, CD5 and CD99, and immature T cells were identified. The above histopathological characteristics and immunohistochemical features supported the diagnosis of type B2 thymoma. However, of note are the numerous HCs with polymorphism and different sizes in thymoma, which instigated the observation of various HCs. Only five cases, including three of type B2 and two of type B3, have been described in the literature (12), to the best of our knowledge. Furthermore, these previous studies had the same aim as that of the present case report, and investigated whether this rare observation maybe a distinct entity or variant of organotypical thymoma WHO B2/B3, and also investigated the missing association between thymoma with an abundance of HCs and thymic carcinoma. However, the results demonstrated that the prevalence of HCs did not herald the association between type B2/B3 thymoma and thymic carcinoma. Finally, the previous studies considered that the corpuscular differentiation was an option for neoplastic cells in organotypical thymoma and may give rise to the predominant histoarchitectural pattern, resulting in a rare distinct variant (12). This was also concluded in the present case. In addition, a previous study demonstrated numerous and large HCs, with highly polymorphic shapes, were identified in human fetuses, and these increased rapidly in number and size at 28 weeks of gestation, prior to subsequently increasing at a decreased rate (1). It was speculated that these HCs changes were also associated with the negative selection process of thymocytes (1). It remains to be elucidated whether a large quantity of HCs in the thymoma may assist with removing apoptotic self-reacting thymocytes. However, the study by Laeng et al (12) detected the apoptotic cells in five cases of thymoma and the results demonstrated that apoptotic cells were prominent in areas of regressive change in all cases. Additionally, the labeling indices of apoptotic cells outnumbered those for proliferating neoplastic cells (12). However, further studies are required to elucidate this molecular mechanism.

Regarding biological behavior, the five cases reported in the literature appeared indolent. By contrast, the tumor in the present study was involved in the lung tissues and right pericardium, although a number of distinctions, including cholesterol crystal, scarring, necrosis and calcium deposits, suggested that it was a long-standing lesion. Furthermore, the tumor wrapped around the superior and inferior vena cava. Thus, the biological behavior of the type of thymoma remains undefined.
Patients such as these should be followed-up regularly. The patient in the present case report began having a severe pain in the right anterior chest 9 months after surgery. It was predicted that the tumor may recur for two reasons. One was that the tumor was invasive, and the other was that the tumor tissue adhering to the pericardium was not resected completely. However, recurrence could not be confirmed as the individual did not agree to undergo an imaging examination.

In conclusion, the type B2 or B3 thymoma abundance of $\mathrm{HCs}$ is rare. Further elucidation is required as to whether it represents a variant of WHO type B2 or B3 thymoma or whether it qualifies as a distinct entity. In addition, it should be differentiated from thymic hyperplasia.

\section{References}

1. Asghar A, Syed YM and Nafis FA: Polymorphism of Hassall's corpuscles in thymus of human fetuses. Int J Appl Basic Med Res 2: 7-10, 2012.

2. Laster AJ, Itoh T, Palker TJ and Haynes BF: The human thymic microenvironment: Thymic epithelium contains specific keratins associated with early and late stages of epidermal keratinocyte maturation. Differentiation 31: 67-77, 1986.

3. Nicolas JF, Reano A, Kaiserlian D and Thivolet J: Epithelial cell heterogeneity in mammalian thymus: Monoclonal antibody to high molecular weight keratins exclusively binds to Hassall's corpuscles. Histochem J 21: 357-364, 1989.

4. Boyd RL, Tucek CL, Godfrey DI, Izon DJ, Wilson TJ, Davidson NJ, Bean AG, Ladyman HM, Ritter MA and Hugo P: The thymic microenvironment. Immunol Today 14: 445-459, 1993.

5. Kuo TT, Mukai K, Eimoto T, Laeng RH, Henry K and Chan JKC: Type A thymoma, in: Travis WD, Brambilla E, Müller-Hermelink HK, Harris CC (Eds.): Tumours of the Lungs, Pleura and Mediastinum. World Health Organisation Classification of Tumours, IARC Press, Lyon, France, pp154-158, 2004.

6. Marino M and Müller-Hermelink HK: Thymoma and thymic carcinoma. Relation of thymoma epithelial cells to the cortical and medullary differentiation of thymus. Virchows Arch 407: 119-149, 1985.

7. Marx A and Müller-Hermelink HK: From basic immunobiology to the upcoming WHO-classification of tumors of the thymus. Pathol Res Pract 195: 515-533, 1999.

8. Müller-Hermelink HK, Marx A and Kirchner T: Advances in the diagnosis and classification of thymic epithelial tumours, In: Anthony PP, MacSween RNM (Eds.): Recent Advances in Histopathology, Churchill Livingstone, Edinburgh, pp49-72, 1994.

9. Pan CC, Chen WY and Chiang H: Spindle cell and mixed spindle/lymphocytic thymomas. An integrated clinicopathologic and immunohistochemical study of 81 cases. Am J Surg Path 25: 111-120, 2001.

10. Quintanilla-Martinez L, Wilkins EW Jr, Choi N, Efird J, Hug E and Harris NL: Thymoma: Histologic subclassification is an independent prognostic factor. Cancer 74: 606-617, 1994.

11. Shimosato $Y$ and Mukai K: Tumors of the mediastinum, in: Rosai J, Sobin LH (Eds.): Atlas of Tumor Pathology, Armed Forces Institute of Pathology, Washington DC, pp93-101, 1997.

12. Laeng RH, Eimoto T, Kuo TT, Zettl A, Marx A, Moschopulos M, Tateyama $\mathrm{H}$ and Shimokawa K: Corpuscularthymoma: Entity or variant of organotypicalthymomas WHO B2/B3? Pathol Res Pract 202: 697-704, 2006.

13. Farr AG, Dooley JL and Erickson M: Organization of thymic medullary epithelial heterogeneity: Implications for mechanisms of epithelial differentiation. Immunol Rev 189: 20-27, 2002.

14. Watanabe N, Wang YH, Lee HK, Ito T, Wang YH, Cao W and Liu YJ: Hassall's corpuscles instruct dendritic cells to induce CD4+CD25+ regulatory $\mathrm{T}$ cells in human thymus. Nature 436: $1181-1185,2005$. 